\title{
56 Gbps Si/GeSi integrated EAM
}

L. Mastronardi, ${ }^{1,}$ M. Banakar, ${ }^{1}$ A.Z. Khokhar, ${ }^{1}$ N. Hattasan, ${ }^{1}$ T. Rutirawut, ${ }^{1}$ T. Domínguez Bucio, ${ }^{1}$ K. M. Grabska, ${ }^{1}$ C. Littlejohns, ${ }^{1,2}$ A. Bazin, ${ }^{1}$ G. Mashanovich, ${ }^{1}$ And F.Y. Gardes ${ }^{1}$

${ }^{1}$ ORC, University of Southampton, University Road Southampton SO17 1BJ, ${ }^{2}$ Silicon Technologies

Centre of Excellence, Nanyang Technological University, 50 Nanyang Avenue, Singapore, 639798

*1.mastronardi@soton.ac.uk

\begin{abstract}
The growing demand for fast, reliable and low power interconnect systems requires the development of efficient and scalable CMOS compatible photonic devices, in particular optical modulators. In this paper, we demonstrate an innovative electro absorption modulator (EAM) developed on an $800 \mathrm{~nm}$ SOI platform; the device is integrated in a rib waveguide with dimensions of a $1.5 \mu \mathrm{m}$ x $40 \mu \mathrm{m}$, etched on a selectively grown GeSi cavity. High speed measurements at $1566 \mathrm{~nm}$ show an eye diagram with dynamic ER of $5.2 \mathrm{~dB}$ at $56 \mathrm{Gbps}$ with a power consumption of $44 \mathrm{fJ} / \mathrm{bit}$.
\end{abstract}

\section{INTRODUCTION}

The modern communication infrastructure can be divided in two categories, the Long-Haul C-band (LHC) and the ShortHaul O-band (SHO) mixed electro-optic systems. LHC advantages derive from the use of single mode fibers (SMF) and high-quality optic fibers amplifiers that allow transmission over hundreds of kilometers without signal regeneration. SHO, on the other end, by implementing cheaper multi-mode fibers (MMF) and optic sources (SLED, VCSEL) is more cost effective, hence, it is vastly used in datacenters.
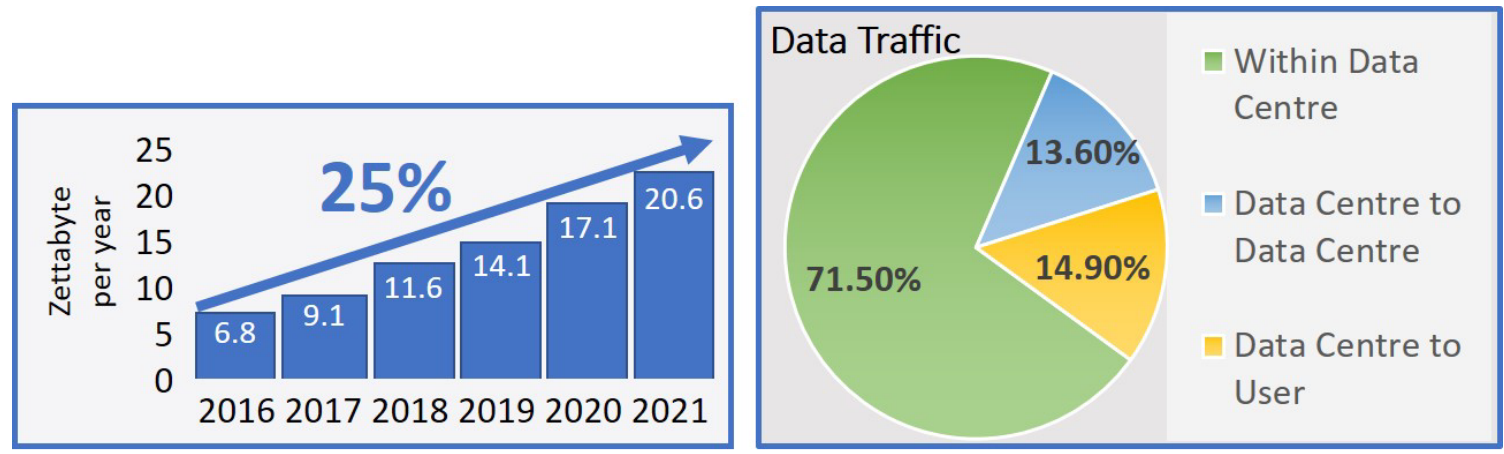

Figure 1 Cisco Annual IP growth Prediction (left) and Data traffic ratios (right) ${ }^{[2]}$.

The SHO bandwidth, however, is limited by the MMF's modal dispersion and represents a bottleneck for aggregate multi wavelength systems with data rate from $200 \mathrm{~Gb} / \mathrm{s}$ and connection distances beyond $500 \mathrm{~m}\left(802.3 \mathrm{bs}\right.$ standard $\left.{ }^{[1]}\right)$. To emphasize further the future scalability requirement needs, a recent Cisco Report ${ }^{[2]}$ has predicted that by 2021 the annual IP traffic will exceed the 20 Zettabyte (left graph of Figure 1), with most of the traffic within the datacenter (right pie chart of Figure 1). In a decade datacenters will require, therefore, link speed approaching $800 \mathrm{~Gb} / \mathrm{s}$ or $1.6 \mathrm{~Tb} / \mathrm{s}$, as envisioned by the Ethernet Alliance's Roadmap ${ }^{[3]}$ (Figure 2). 
Return to the Manage Active Submissions page at http://spie.org/submissions/tasks.aspx and approve or disapprove this submission. Your manuscript will not be published without this approval. Please contact author_help@spie.org with any questions or concerns.

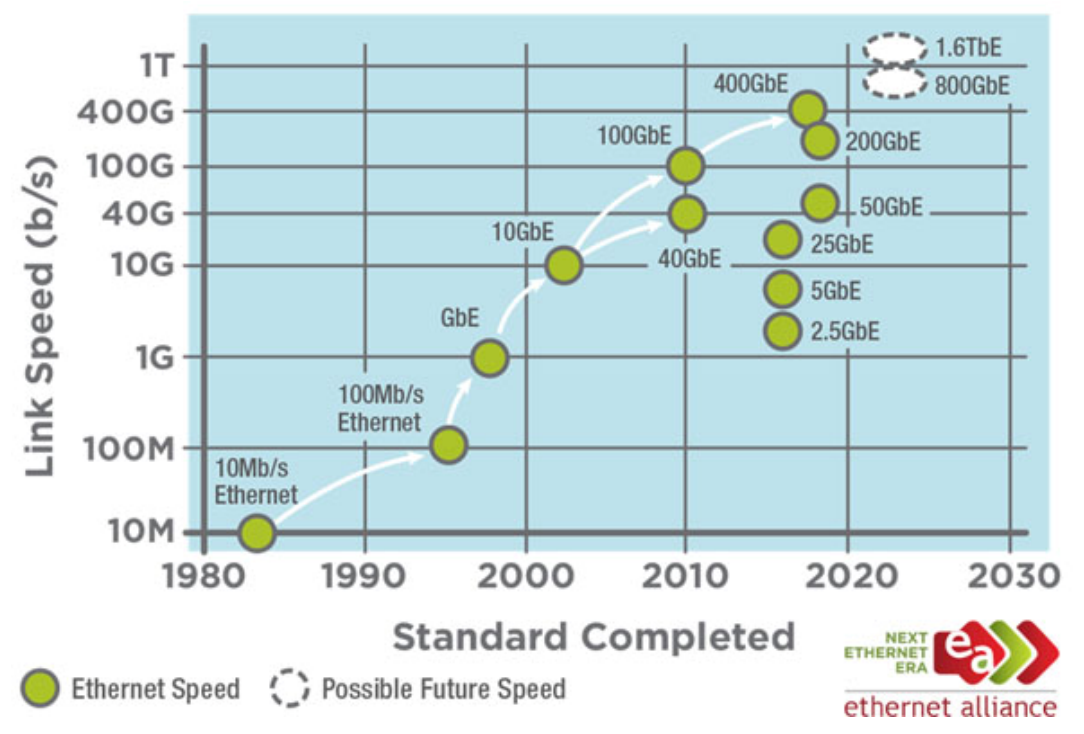

Figure 2 Ethernet Alliance's roadmap for the Terabit Ethernet ${ }^{[3]}$

That is why companies are investing in reduction of single mode fibers (SMF) deployment $\operatorname{costs}^{[4]}$ and integrating Silicon Photonic Circuits (Si-PIC) into transceivers ${ }^{[5]}$ to boost the bandwidth in datacenters. Si-PICs can, indeed, reduce the power consumption of transceivers through high-density integration, increase data rate for long short to mid-range distance and reduce latency by avoiding conversion from/to electric signals and reduce the use of copper wires. Compatible materials for this technology are based on group IV compounds, which exploit the CMOS knowhow and do not require the conversion of exiting fabrication facilities, making Silicon Photonics very attractive to the industry. So far, integrated silicon and multilayer based systems ${ }^{[6]-[8]}$ including high speed photodetectors ${ }^{[9],[10]}$, wavelength division multiplexing (WDM) filters ${ }^{[11]}$ and in particular optical modulators ${ }^{[12]-[13]}$ have been successfully demonstrated.

In silicon, Plasma Dispersion Effect ${ }^{[14]-[17]}$ based modulators achieve high speed but at the expense of a relatively large footprint $\left(\sim \mathrm{mm}^{2}\right)$ and power consumption (pJ/bit) and this makes high density integration challenging. To alleviate those limitations highly resonant devices have been developed ${ }^{[18],[19]}$. However, these devices have limited operational optical

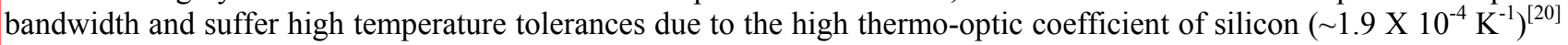
and the wavelength selectivity of ring resonators.

Through the use of Ge and GeSi, Electro Absorption Effect based modulators ${ }^{[21]-[23]}$ (Franz-Keldysh Effect ${ }^{[24],[25]}$ in bulk for the C/L-band and the Quantum Confined Stark Effect ${ }^{[26]}$ in quantum well materials for the $\mathrm{O}$ to $\mathrm{C}$ Band) offer the best trade-offs in terms of speed, footprint and power consumption for highly integrated Si-PICs and short/medium-haul applications. For bulk FK based modulators, Thermal Anneal ${ }^{[27]}$ (RTA) or Rapid Melt Growth ${ }^{[28],[29]}$ (RMG) techniques can be used to integrate arrays of modulators working at different bandgap energy or wavelengths by tuning of the material composition. These techniques offer the potential to overcome the limited optical bandwidth $(\sim 35 \mathrm{~nm})$ of this class of modulators.

Here, we present an integrated rib waveguide modulator ${ }^{[30]}$ realized on a selectively grown $\mathrm{Si} / \mathrm{GeSi}$ cavity on $800 \mathrm{~nm}$ SOI wafer; the active area is a wrap-around PIN hetero-structure with dimension $1.5 \mu \mathrm{m} \times 40 \mu \mathrm{m}$, which enables electric field independency from the rib width and the possibility to tailor the waveguide dimension to provide better optical mode confinement and propagation for both polarizations. High speed measurements show a dynamic ER of $5.2 \mathrm{~dB}$ at a data rate of $56.2 \mathrm{Gbps}$, power consumption of $44 \mathrm{fJ} / \mathrm{bit}$ and modulation bandwidth of $56 \mathrm{GHz}$. This design, therefore, provides a highly customizable and simple platform for compact-high-speed electro absorption modulators.

\section{MODULATOR DESIGN}

Simulations were carried to optimize the design and develop the process for integrating a vertical PIN diode in a standard rib waveguide. The platform used for simulations is summarized in the graph of Figure 3, upon defining a set of parameters such as rib width, doping levels and distances, the cross-section process first and the electric field distribution after, are simulated (yellow squares, S1 and S2). By extrapolating the electric field map, the absorption coefficient due to 


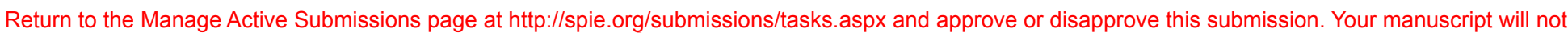
be published without this approval. Please contact author_help@spie.org with any questions or concerns.

FKE is calculated (material simulation) by using a mathematical model ${ }^{[31]}$, then, the complex refractive index in the cross-section mesh is retrieved with the Kramers-Kronig relations ${ }^{[32]}(\mathrm{M} 1)$. The electric and the material simulations are finally used in the mode solver (M2) to find the optic mode distribution and calculate the Insertion Loss (IL), the Extinction Ratio (ER), the device speed and power consumption.



Figure 3 Simulation Platform Schematics

After optimizations, a wrap-around PIN Si/GeSi hetero-structure integrated in a $1.5 \mu \mathrm{m}$ wide rib waveguide has been adopted, this is shown in the left picture of Figure 4. The P doping of the diode is defined in a $100 \mathrm{~nm}$ thick silicon layer (light brown), whereas the $600 \mathrm{~nm}$ thick intrinsic region comprehends the Ge buffer layer (black) and a GeSi area (purple). The $\mathrm{N}$ doping (orange/red area) is, instead, defined on the top and right side of the rib with a thickness of $\sim 100$ $\mathrm{nm}$. The advantages of this approach can be seen in the simple process and the independency of the electric field strength from the rib width which allows to realize wide waveguides. The high customizable design permits, also, the device to support either TE or TM modes (in this first run TE mode has been chosen) but more importantly to confine better the optical mode. In the right picture of Figure 4, the overlap between the optical mode and the electric distribution is depicted, showing an electric field of about $40 \mathrm{kV} / \mathrm{cm}$ where the optical mode is mostly confined.

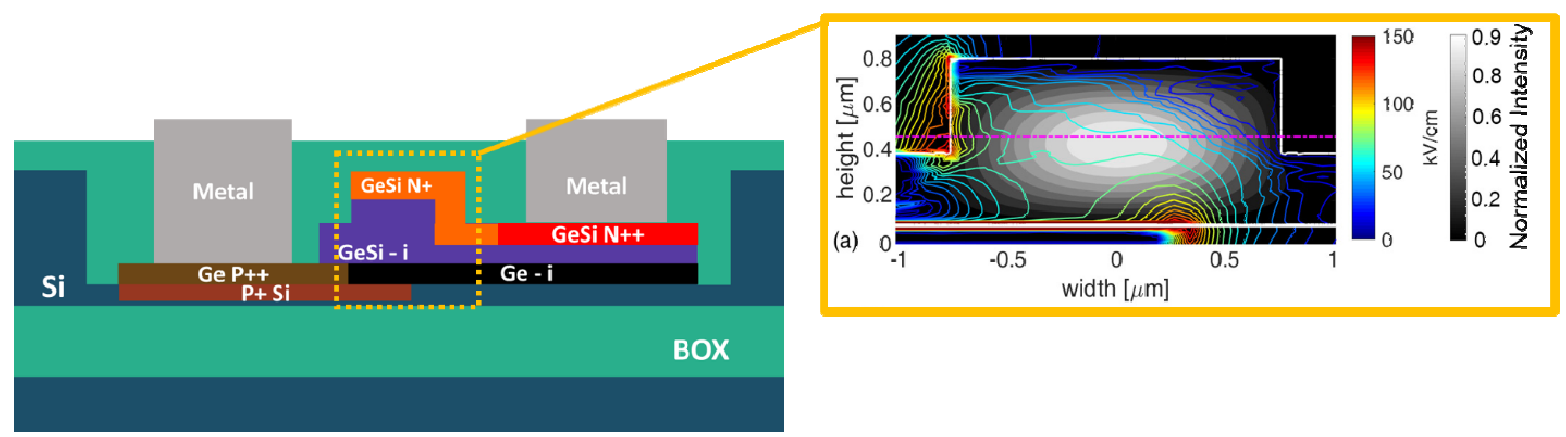

Figure 4 (Left) cross-section design. (Inset) electric field and optical mode overlap and distributions; in rainbow the electric field strength in $\mathrm{kV} / \mathrm{cm}$, instead in grey tone the normalized optical power of the propagating TE mode.

\section{FABRICATION}

Once the design has been fixed, the process is implemented with the fabrication steps depicted in Figure 5. The fabrication process requires three etches and four implantations. 
Return to the Manage Active Submissions page at http://spie.org/submissions/tasks.aspx and approve or disapprove this submission. Your manuscript will not be published without this approval. Please contact author_help@spie.org with any questions or concerns.

a)

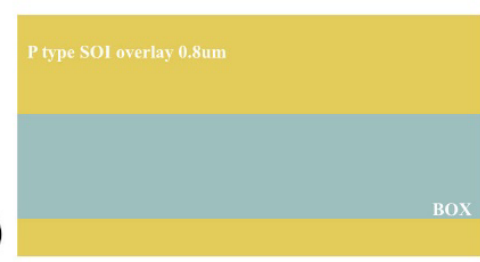

d)



g)

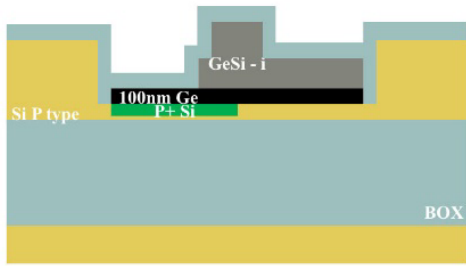

j)

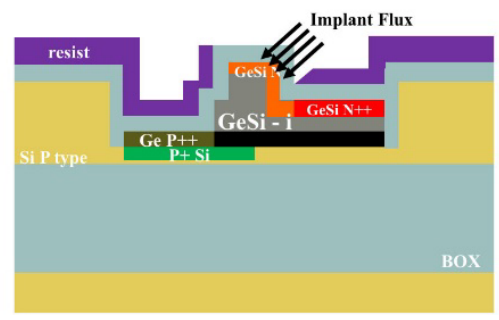

b)

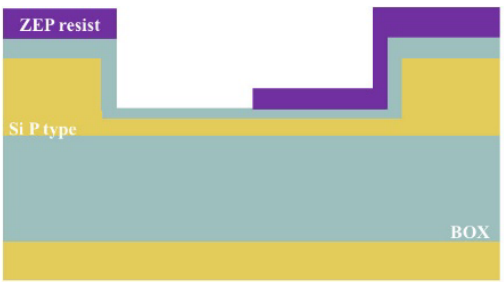

e)

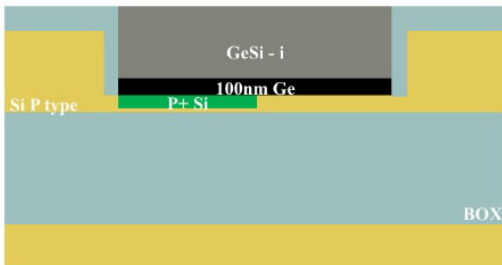

h)



k)

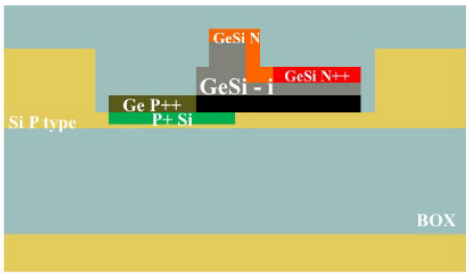

Figure 5 Principal Process Steps c)



f)



i)

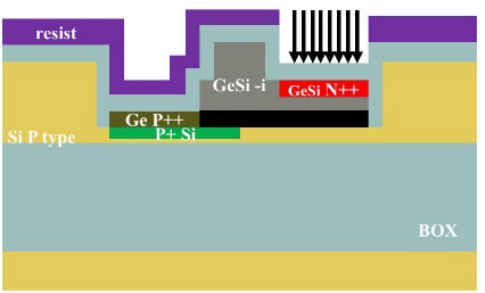

l)

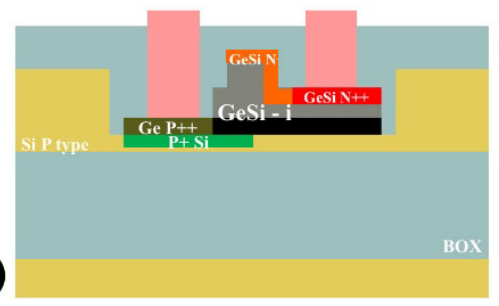

The SOI wafer used for the fabrication of this device has an $800 \mathrm{~nm}$ thick Si overlayer; GeSi epitaxy cavities with a size of $50 \mu \mathrm{m} \times 40 \mu \mathrm{m}$ are etched with a depth of $700 \mathrm{~nm}$ leaving approximately $100 \mathrm{~nm}$ of Si where the P side of the vertical PIN structure is realized using Boron implantation with a concentration of $\sim 10^{18} \mathrm{~cm}^{-3}$ (Figure $5 \mathrm{at}$ to $\mathrm{c}$ ). After doping, the cavity is filled with GeSi using a two steps selective epitaxial growth; first $\sim 100 \mathrm{~nm}$ of Ge buffer layer is deposited in the cavity trench, then a uniform GeSi layer of about $600 \mathrm{~nm}$ thickness is deposited. Chemical Mechanical Polishing, to remove any GeSi excess and planarize the wafer, precedes the waveguide etch step (Figure $5 \mathrm{~d}$, e) that is defined by a two etch process. In the first step (f) only the left side of the cavity is etched with a depth of $200 \mathrm{~nm}$, then the waveguide is etched to a depth of about $400 \mathrm{~nm}$ to realize the rib as shown in Figure $5 \mathrm{~g}$. On the left side of the rib only $100 \mathrm{~nm}$ of $\mathrm{Ge}$ is left, whereas on the right side of the rib, a $300 \mathrm{~nm}$ thick slab is obtained. The waveguide etch technique adopted is self-aligned because the $\mathrm{Si}$ and the GeSi waveguides are etched on the same time (Figure 6, picture on the left), reducing interface mode mismatch. The simulated interface optical loss is about $0.3 \mathrm{~dB}$ per facet. During the waveguide definition, coupling gratings at the waveguide extremities and silicon normalization waveguides close to the device waveguide are, also, defined.

High dose ion implantations to define the ohmic contacts, are then performed. On the left side slab, $\mathrm{BF}_{2}(\mathrm{P}++)$ with a concentration of $\sim 10^{20} \mathrm{~cm}^{-3}$ is implanted in the thin Ge layer (Figure $5 \mathrm{~h}$ ); on the slab on the right (Figure $5 \mathrm{i}$ ), the GeSi layer is doped with a $\sim 10^{19} \mathrm{~cm}^{-3}$ concentration of Phosphorus $(\mathrm{N}++)$. The doping to define the $\mathrm{N}$ side of the PIN junction is realized on the top and right side of rib with an angled implantation $\left(45^{\circ}\right)$ using Phosphorus with a concentration of $\sim 10^{18} \mathrm{~cm}^{-3}$ and an estimated implantation depth of $100 \mathrm{~nm}$ (Figure $5 \mathrm{j}$ ). Finally, Rapid Thermal Anneal is performed to activate the doping. The oxide deposition, VIA definition and metal deposition are shown in Figure 5 (k, 1); in Figure 6, instead, the top view (left) and the FIB cut (right) of the realized device are shown. 
Return to the Manage Active Submissions page at http://spie.org/submissions/tasks.aspx and approve or disapprove this submission. Your manuscript will not be published without this approval. Please contact author_help@spie.org with any questions or concerns.
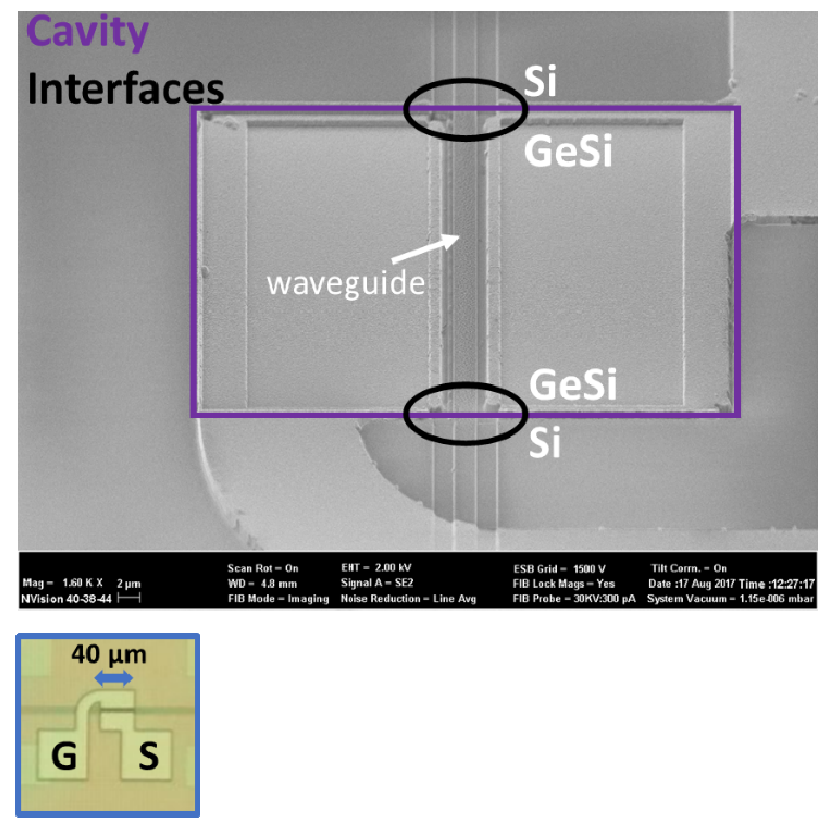

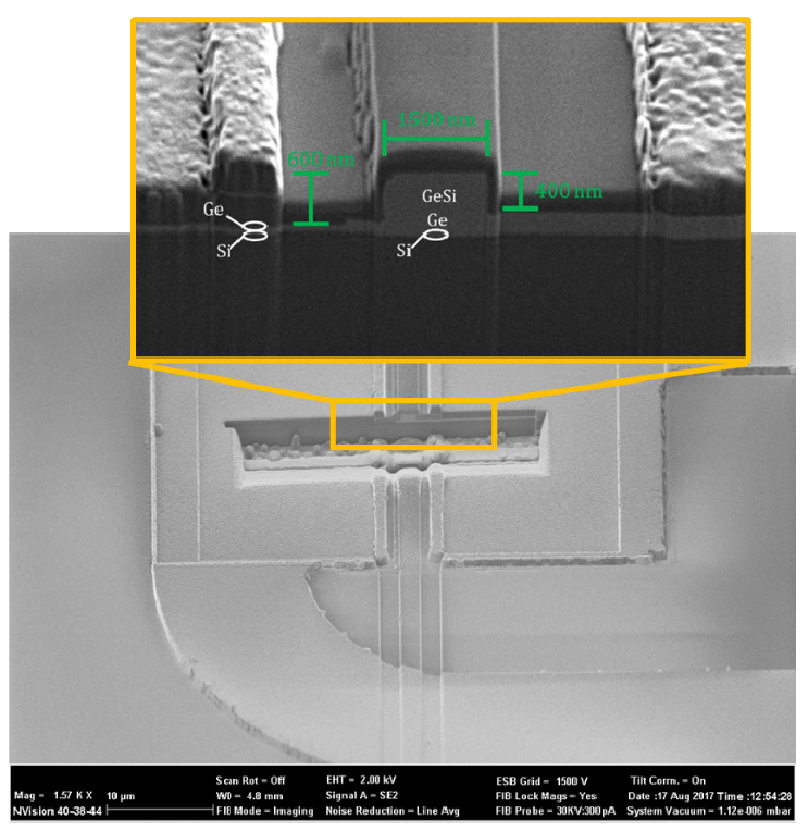

Figure 6 Top view and FIB cut (yellow inset) of the realized device

\section{DC AND HIGH-SPEED MEASUREMENTS}

To assess the electro-optic effect, DC measurements are performed; for this purpose, a semi-automatic setup (diagram in Figure 7) is built using the Agilent 8163B Lightwave Multimeter with laser module 81949A and Power Meter 81630B, the Keysight 2400 source meter and tungsten tip DC probes from Cascade Microtech. The setup is controlled with Matlab routines.

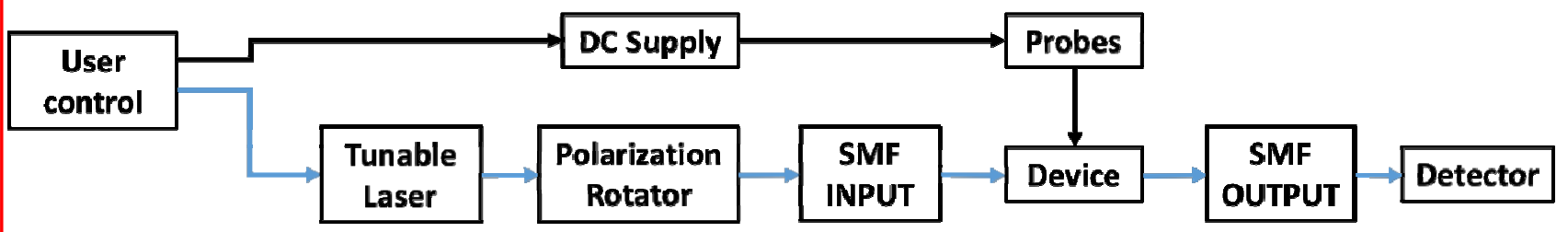

Figure 7 DC Measurements Bench Schematics

The IV curve has been measured to assess the electric performances of the PIN diode and measure the dark current at reverse biases. Mainly caused by surface current and defects in the crystal, dark current affects the device performances by increasing the power consumption. To limit the dark current, a passivation layer is deposited before metallization to prevent current surface paths. The measured IV is shown in Figure 8, at $-4 \mathrm{~V}$ the dark current is about $10 \mu \mathrm{A}$, better epitaxial growth and the passivation layer would lead to its reduction. 
Return to the Manage Active Submissions page at http://spie.org/submissions/tasks.aspx and approve or disapprove this submission. Your manuscript will not be published without this approval. Please contact author_help@spie.org with any questions or concerns.

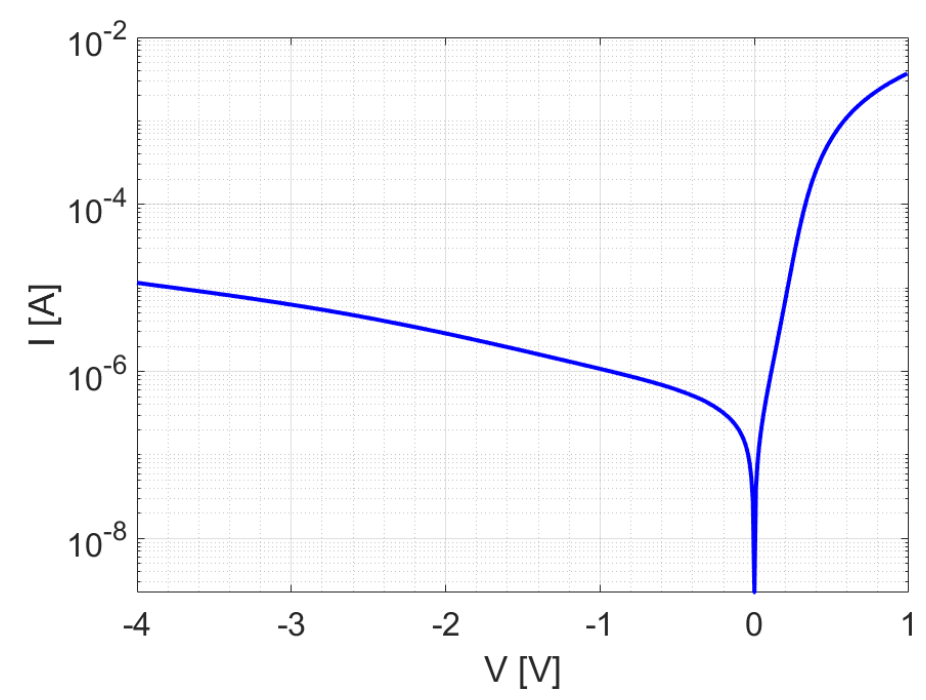

Figure 8 IV curve

The electro-absorption effect, is then tested by measuring optical transmission spectra while applying DC biases. The resulting cavity Insertion Loss (IL) and Extinction Ratio (ER) are shown in Figure 9. The IL measures the optical loss between the laser input and the detector, hence it comprehends coupling loss, propagation loss, losses at the Si-GeSi interfaces and material absorption in the cavity. By using the silicon normalization waveguides and estimating, through simulation, the losses at the Si-GeSi interfaces it is possible to retrieve the IL of the cavity, dominated by material absorption. For this first prototype the presence of the Ge buffer layer is responsible for a higher IL, due to background and undesirable absorption in the wavelength range of interest. Insertion Loss can be improved by using Rapid Thermal Anneal ${ }^{[27]}$ before the device is realized to diffuse silicon into the Ge buffer layer to obtain a homogenous GeSi layer, or growing GeSi layer with epitaxial techniques that do not require any Ge buffer layer ${ }^{[28],[29]}$.

However, the ER, measured by the absorption difference between the high and low states, reaches considerable values and shows a secondary peak for wavelength above $1570 \mathrm{~nm}$ confirming the effect of the Ge buffer layer. For a reverse bias of $1 \mathrm{~V}$ the ER is $3 \mathrm{~dB}$ at $1540 \mathrm{~nm}$, at higher reverse biases it increases up to $7.5 \mathrm{~dB}$ (bias $=-4 \mathrm{~V}$ ); an incremental ER rate of about $1.5 \mathrm{~dB} / \mathrm{V}$ is found around $1540-1545 \mathrm{~nm}$.

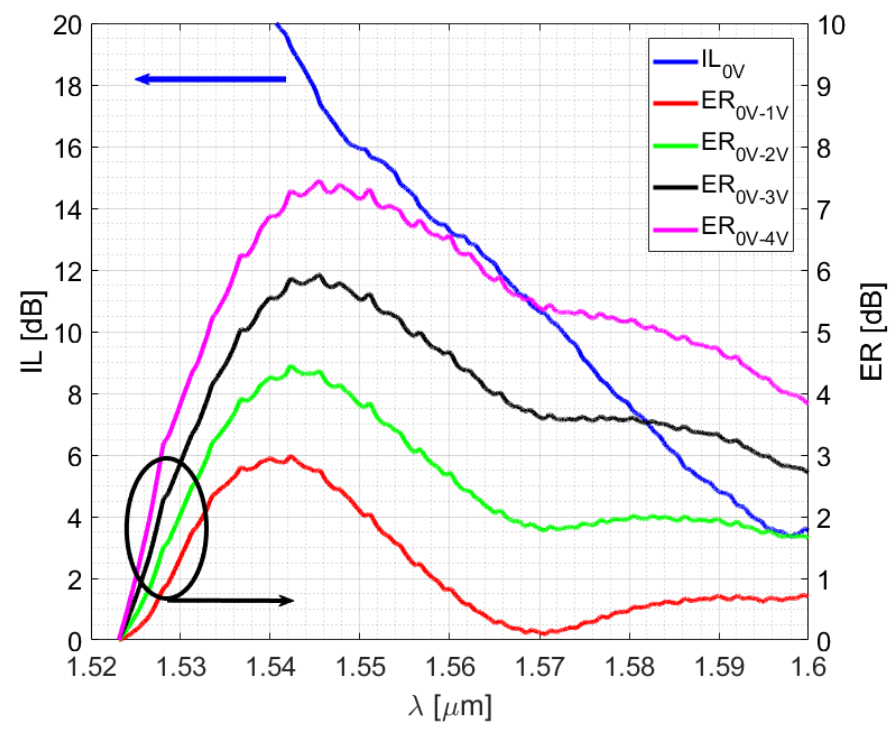

Figure 9 (left) IL and ER, (right) for different reverse biases 


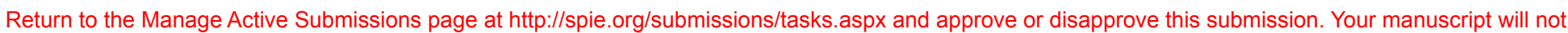
be published without this approval. Please contact author_help@spie.org with any questions or concerns.

High-speed measurements are conducted by including in the DC setup a pseudorandom binary sequence (PRBS) generator, RF amplifier and attenuators, an EDFA, an optical filter and a DCA as shown in Figure 10. The PRBS is coupled to the RF amplifier and the in-line RF attenuators to generate the electric bitstream with the required voltage swing; the DC and RF electrical signals are, then, mixed with a bias-T and fed into the modulator using GS probes not50-ohms terminated. At the modulator output, the low noise EDFA and bandpass filter are used to amplify the modulated optical signal, which is finally analyzed by the DCA.



Figure 10 High-Speed Measurement Bench Schematics

Several tests are conducted to find the electro-optic parameters that offer the best performances; first, measurements to investigate the maximum speed supported by the device are performed, then by fine tuning the parameters the highest dynamic ER at maximum speed is found. It must be noted that, the probes were not resistively loaded and caused RF reflections at the modulator ports increasing the Vpp. The optical bandwidth of the EDFA and optical filter used in the setup also limited the measurement of the modulation at a wavelength of $1570 \mathrm{~nm}$.

By choosing a modulation wavelength of $1566 \mathrm{~nm}$ and applying a bias of $-2.7 \mathrm{~V}$ and $2.2 \mathrm{Vpp}$, an ER of $5.2 \mathrm{~dB}$ at 56.2 Gbps is recorded (Figure 11), which represents the best performance of this first prototype.
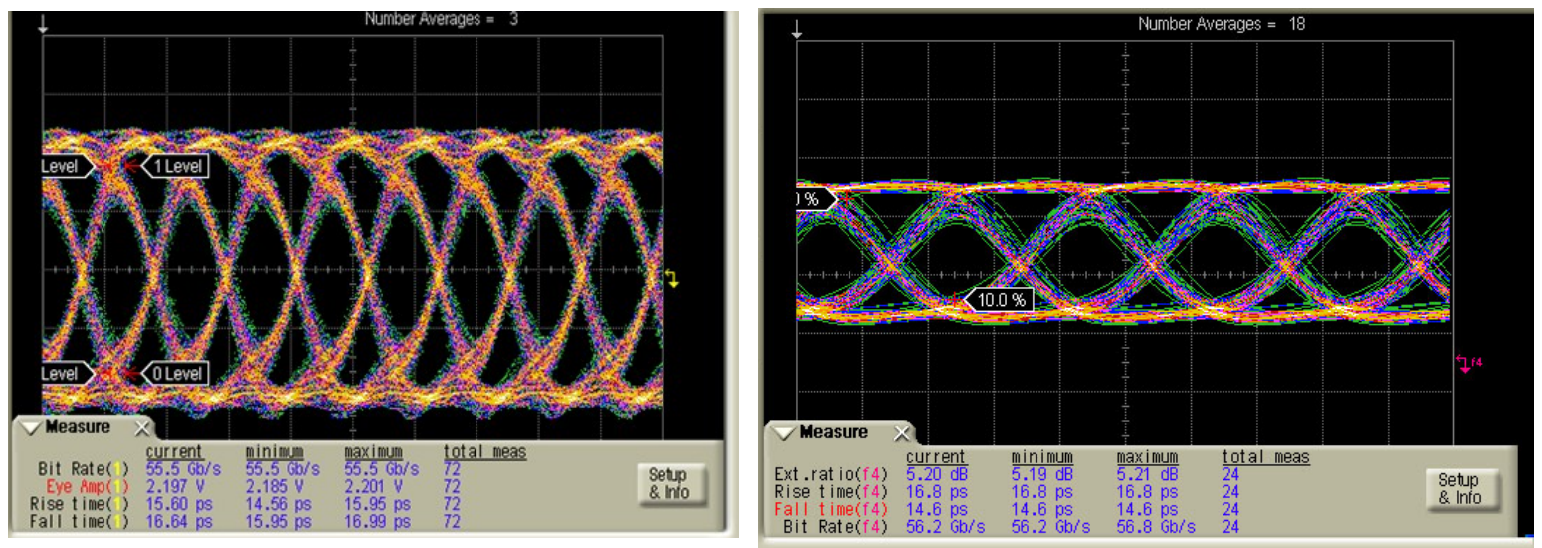

Figure 11 (Left) Electrical Eye at 56 Gbps and 2.2 Vpp. (Right) Output optical eye with a measured dynamic ER of 5.2 $\mathrm{dB}$ at 56.2 Gbps at wavelength of $1566 \mathrm{~nm}$

The maximum speed recorded is, however, not limited by the device but by the setup used. In fact, the EO bandwidth, estimated as the reciprocal of the device rise time: $\tau_{r}=\sqrt{\left(\tau_{o}^{2}-\tau_{e}^{2}\right)}$ (with $\tau_{\mathrm{o}}$ the rise time of the optical eye and $\tau_{\mathrm{e}}$ the rise time of the electrical input eye) is found to be $\sim 56 \mathrm{GHz}$, making possible rate speed higher than 56 Gbps by using NRZ modulation.

The last set of measurements investigated the EAM electric equivalent circuit (Figure 12) by mean of S11 measurements and numerical fitting. 
Return to the Manage Active Submissions page at http://spie.org/submissions/tasks.aspx and approve or disapprove this submission. Your manuscript will not be published without this approval. Please contact author_help@spie.org with any questions or concerns.

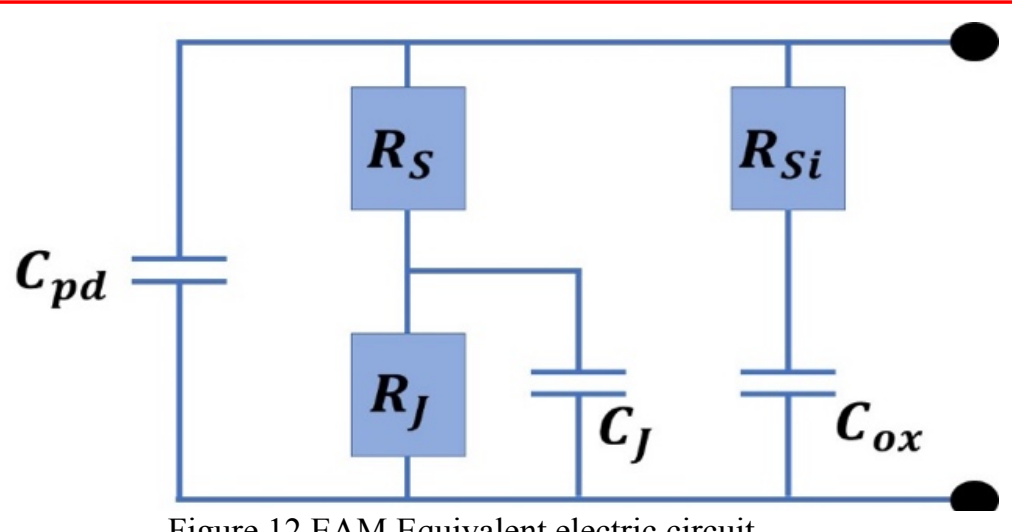

Figure 12 EAM Equivalent electric circuit

The device is modelled with six lumped elements, the capacitance due to the metallic pads $\mathrm{C}_{\mathrm{pd}}$, the series resistance $\mathrm{R}_{\mathrm{S}}$, the resistance $\mathrm{R}_{\mathrm{J}}$ and capacitance $\mathrm{C}_{\mathrm{J}}$ of the PIN junction, the BOX capacitance $\mathrm{C}_{\mathrm{ox}}$ and the substrate resistance $\mathrm{R}_{\mathrm{Si}}$. The fitted values are shown in Table 1.

Table 1 Equivalent lumped elements fitted with S11 measurements

\begin{tabular}{|c|c|c|c|c|c|}
\hline $\begin{array}{c}\mathbf{C}_{\mathbf{p d}} \\
(\mathbf{f F})\end{array}$ & $\begin{array}{c}\mathbf{R}_{\mathbf{S}} \\
\mathbf{( \Omega )}\end{array}$ & $\begin{array}{c}\mathbf{R}_{\mathbf{J}} \\
\mathbf{( \Omega )}\end{array}$ & $\begin{array}{c}\mathbf{C}_{\mathbf{J}} \\
(\mathbf{f F})\end{array}$ & $\mathbf{C}_{\mathbf{0 x}}$ & $\mathbf{\mathbf { R } _ { \mathbf { S i } }}$ \\
$\mathbf{( f F )}$ & $\mathbf{( \Omega )}$ \\
\hline 5 & 150 & 1500 & 11 & 30 & 350 \\
\hline
\end{tabular}

The power of the EAM is, then, found to be $\mathrm{C}_{\mathrm{J}} \mathrm{Vpp}^{2} / 4=44 \mathrm{fJ} / \mathrm{bit}$ at $56 \mathrm{Gbps}$, considering the increased $\mathrm{Vpp}(\sim 4 \mathrm{~V})$ due to $R F$ reflections caused by not terminated RF probes.

\section{CONCLUSION}

A high-speed low power consuming and compact footprint $\left(60 \mu^{2}\right)$ GeSi EAM on an $800 \mathrm{~nm}$ SOI platform has been designed, fabricated and characterized. The novel vertical PIN diode allowed the realization of an integrated rib modulator with a height-to-width ratio of $\sim 2.4$, permitting a better optical confinement and tolerant design parameters due to the independency of the electric field strength from the rib width. Successfully working at $1566 \mathrm{~nm}$, the demonstrated rate speed of 56.2 Gbps with dynamic ER of $5.2 \mathrm{~dB}$ is only limited by the setup, in fact an EO bandwidth of $56 \mathrm{GHz}$ is calculated. Finally, S11 measurement and fitting revealed a power consumption of about $44 \mathrm{fJ} / \mathrm{bit}$. Therefore, this concept demonstrated to be a viable solution to integrate optic switches in a CMOS photonic circuits for short reach communication in the $\mathrm{C}$ and $\mathrm{L}$ bands.

\section{FUNDING AND ACKNOWLEDGMENTS}

This work was funded by EPSRC First Grant (EP/K02423X/1), EPSRC Platform Grant (EP/N013247/1) and H2020 project COSMICC (688516), EPSRC Prosperity Partnership (EP/R003076/1).

This work had support from the Optoelectronic research Centre (ORC) and the Southampton nanofabrication centre. CGL acknowledges support from National Research Foundation of Singapore (NRFCRP12-2013-04).

The data from this work is available online in the repository at https://doi.org/10.5258/SOTON/D0354

Published by Society of Photo Optical Instrumentation Engineers (SPIE) under the terms of the Creative Commons Attribution 4.0 License. Further distribution of this work must maintain attribution to the author(s) and the published article's title, journal citation, and DOI. 
Return to the Manage Active Submissions page at http://spie.org/submissions/tasks.aspx and approve or disapprove this submission. Your manuscript will not be published without this approval. Please contact author_help@spie.org with any questions or concerns.

\section{REFERENCES}

[1] IEEE Standard for Ethernet - Amendment 10: Media Access Control Parameters, Physical Layers, and Management Parameters for $200 \mathrm{~Gb} / \mathrm{s}$ and $400 \mathrm{~Gb} / \mathrm{s}$ Operation," in IEEE Std 802.3bs-2017 (Amendment to IEEE $802.3-2015$ as amended by IEEE's $802.3 \mathrm{bw}-2015$, 802.3by-2016, 802.3bq-2016, 802.3bp-2016, 802.3br2016, 802.3bn-2016, 802.3bz-2016, 802.3bu-2016, 802.3bv-2017, and IEEE 802.3-2015/Cor1-2017), vol., no., pp.1-372, (2017).

[2] Cisco, "Cisco visual networking index: Forecast and methodology," 2015-2020 (2016).

[3] Ethernet Alliance, "The 2018 Ethernet Roadmap," https://ethernetalliance.org/the-2018-ethernet-roadmap/

[4] Morgan, T. P., "Datacenters to Get A High Fiber Bandwidth Diet," The Next Platform (2016).

[5] Offrein, B. J., "Silicon photonics for the datacenter," Optical Fiber Communications Conference and Exhibition (OFC), 1-3 (2015).

[6] R. Soref. "The past, present, and future of silicon photonics," IEEE Journal of Selected Topics in Quantum Electronics 12(6), 1678- 1687 (2006).

[7] Chen, Y., Domínguez Bucio, T., Khokhar, A.Z., Banakar, M., Grabska, K., Gardes, F.Y., Halir, R., MolinaFernández, Í., Cheben, P., He, J.-J., 2017. Experimental demonstration of an apodized-imaging chip-fiber grating coupler for Si3N4 waveguides. Optics Letters 42, 3566-3569 (2017).

[8] Dominguez Bucio, T., Khokhar, A.Z., Lacava, C., Stankovic, S., Mashanovich, G.Z., Petropoulos, P., Gardes, F.Y., "Material and optical properties of low-temperature NH3-free PECVD SiNxlayers for photonic applications," Journal of Physics D: Applied Physics 50 (2017).

[9] Chaisakul, P., Marris-Morini, D., Isella, G., Chrastina, D., Rouifed, M.S., Le Roux, X., Edmond, S., Cassan, E., Coudevylle, J.R., and Vivien, L., "10-Gb/s Ge/SiGe multiple quantum-well waveguide photodetector," IEEE Photonics Technology Letters 23, 1430-1432 (2011).

[10]Littlejohns, C., Hu, Y., Gardes, F. Y., Thomson, D. J., Reynolds, S., Mashanovich, G. Z. and Reed, G. T., "50 $\mathrm{Gb} / \mathrm{s}$ silicon photonics receiver with low insertion loss," IEEE Photonics Technology Letters, 26 (7), 714-717 (2014).

[11] Hu, Y., F. Y. Gardes, D. J. Thomson, G. Z. Mashanovich, and G. T. Reed. 2013. "Interleaved Angled MMI CWDM Structure on the SOI Platform." In IEEE International Conference on Group IV Photonics GFP, 21-22.

[12] Reed, G. T., Mashanovich, G., Gardes, F. Y. and Thomson, D. J., "Silicon optical modulators," Nat Photon. 4(8), 518-526 (2010).

[13] Chaisakul, P., Marris-Morini, D., Rouifed, M., Frigerio, J., Chrastina, D., Coudevylle, J., Le Roux, X., J. R., Edmond, S., Isella, G., and Vivien, L., "Recent progress in GeSi electro-absorption modulators," 2014 Sci. Technol. Adv. Mater. 15014601.

[14] Debnath, K., Thomson, D., Zhang, W., Khokhar, S., Littlejohns, C., Byers, J., Mastronardi, L., Husain, M., Gardes, F., Reed, G. and Saito, S., "20Gbps silicon lateral MOS-Capacitor electro-optic modulator," in CLEO: Science and Innovations 2018. OSA Publishing. 2 pp, pp. 1-2.

[15] Debnath, K., Thomson, D., Zhang, W., Khokhar, S., Littlejohns, C., Byers, J., Mastronardi, L., Husain, M., Ibukuro, K., Gardes, F., Reed, G. and Saito, S., "All-silicon carrier accumulation modulator based on a lateral MOS-capacitor," Photonics Research, 6 (5), 373-379 (2018).

[16] Reed, G. T., Thomson, D. J., Gardes, F. Y., Hu, Y., Fedeli, J.-M. and Mashanovich, G. Z., "High-speed carrierdepletion silicon Mach-Zehnder optical modulators with lateral PN junctions," Frontiers in Physics, vol. 2, 77, (2014).

[17] Thomson, D. J., Gardes, F. Y., Fedeli, J.-M., Zlatanovic, S., Hu, Y., Kuo, B. P. P., Myslivets, E., Alic, N., Radic, S., Mashanovic, G., Reed, G. T., "50-Gb/s Silicon Optical Modulator," in IEEE Photonics Technology Letters, 24(4), 234-236 (2012).

[18] Gardes, F. Y., A. Brimont, P. Sanchis, G. Rasigade, D. Marris-Morini, L. O’Faolain, F. Dong, et al. 2009. "High-Speed Modulation of a Compact Silicon Ring Resonator." In IEEE International Conference on Group IV Photonics GFP, 241-43.

[19] Debnath, K., O’Faolain, L., Gardes, F.Y., Steffan, A.G., Reed, G.T., Krauss, T.F., 2012. Cascaded modulator architecture for WDM applications. Optics Express 20, 27420.

[20] Cocorullo, G., Della Corte, F. G., Rendina, I., "Temperature dependence of the thermo-optic coefficient in crystalline silicon between room temperature and $550 \mathrm{~K}$ at the wavelength of $1523 \mathrm{~nm}$," Appl. Phys. Lett. Lett. 74, 3338 (1999). 
Return to the Manage Active Submissions page at http://spie.org/submissions/tasks.aspx and approve or disapprove this submission. Your manuscript will not be published without this approval. Please contact author_help@spie.org with any questions or concerns.

[21]Feng, D., Qian, W., Liang, H., Kung, C. C., Zhou, Z., Li, Z., Levy, J. S., Shafiiha, R., Fong, J., Jonathan Luff, B., and Asghari, M., "High-speed GeSi electroabsorption modulator on the SOI waveguide platform," IEEE Journal of Selected Topics in Quantum Electronics 19(6), 64-73 (2013).

[22] Srinivasan, S. A., Pantouvaki, M., Gupta, S., Chen, H. T., Verheyen, P., Lepage, G., Roelkens, G. Saraswat, K., Thourhout, D. V., Absil, P., and Campenhout, J. V., "56 Gbps germanium waveguide electro-absorption modulator," Journal of Lightwave Technology 34(2), 419-424 (2016).

[23] Rouifed, M., Chaisakul, P., Marris-Morini, D., Frigerio, J., Isella, G., Chrastina, D., Edmond, S., Roux, X., Coudevylle, J., and Vivien, L., "Quantum-confined Stark effect at $1.3 \mu \mathrm{m}$ in $\mathrm{Ge} / \mathrm{Si} 0.35 \mathrm{Ge} 0.65$ quantum-well structure," Opt. Lett. 37, 3960-3962 (2012).

[24] W. Franz, "Einfluß eines elektrischen feldes auf eine optische Absorptionskante," Zeitschrift Naturforschung Teil A 13, 484- 489 (1958).

[25] L. V. Keldysh, "Behavior of non-metallic crystals in strong electric fields," Soviet Journal of Experimental and Theoretical Physics 6, 763 (1958).

[26] Kuo, Y.-H., Lee, Y. K., Ge, Y., Ren, S., Roth, J. E., Kamins, T. I., Miller, D. A. B. and Harris, J. S., "Strong quantum-confined Stark effect in germanium quantum-well structures on silicon," Nature volume 437, pages 1334-1336 (2005).

[27] Mastronardi, L., Banakar, M., Khokhar, A.Z., Dominguez Bucio, T., Littlejohns, C.G., Bernier, N., Robin, E., Rouvière, J.-L., Dansas, H., Gambacorti, N., Mashanovich, G.Z., Gardes, F.Y., "SiGe bandgap tuning for high speed earn," in ECS Transactions of 231st ECS Meeting, 77(6), 59-63 (2017).

[28] Littlejohns, C. G., Nedeljkovic, M., Mallinson, C. F., Watts, J. F., Mashanovich, G. Z., Reed, G. T., \& Gardes, F. Y., "Next Generation Device Grade Silicon-Germanium on Insulator", Scientific Reports, 5, 8288 (2015).

[29] Littlejohns, C. G., Dominguez Bucio, T., Nedeljkovic, M., Wang, H., Mashanovich, G. Z., Reed, G. T., \& Gardes, F. Y., "Towards a fully functional integrated photonic-electronic platform via a single SiGe growth step," Scientific Reports, 6, 19425 (2016).

[30] Mastronardi, L., Banakar, M., Khokhar, A. Z., Hattasan, N., Rutirawut, T., Domínguez Bucio, T., Grabska, K. M., Littlejohns, C., Bazin, A., Mashanovich, G., and Gardes, F. Y., "High-speed Si/GeSi hetero-structure Electro Absorption Modulator," Opt. Express 26, 6663-6673 (2018).

[31] Liu, J., "Gesi photodetectors and electro-absorption modulators for Si electronic-photonic integrated circuits," Massachusetts Institute of Technology (2007).

[32] Kronig, R. de L., "On the theory of the dispersion of X-rays", J. Opt. Soc. Am. 12 (6), 547 (1926). 